\title{
Proceeding
}

Supplementary Issue: Spring Conferences of Sports Science. Costa Blanca Sports Science Events, 14-15 June 2019. Alicante, Spain.

\section{An empirical study for assessing the level of enjoinment in an Italian primary school}

\author{
SALVATORE PIGNATO ${ }^{1}$, RACHELE BONOMO1 ${ }^{1}$, ROBERTO COPPOLA ${ }^{1}$, FRANCESCO CASOLO ${ }^{2}$ \\ ${ }^{1}$ Faculty of Human and Society Sciences, University of Enna «Kore», Italy \\ ${ }^{2}$ "Cattolica» University of Milan, Italy
}

\begin{abstract}
It is well established the relationship between the enjoyment within the physical activities and the personal motivation to follow up a healthy lifestyle. Therefore, the aim of this study was to assess the level of enjoinment during and after the practice of physical and sport activities in school sector. The sample was recruited from a primary school in the south of Italy and the participants had the following characteristics: male: 75; female: 75; mean age: 9.45. The participants were divided into two groups: control group (children did not practice any physical activities) and experimental group (children performed structured physical and sport activities). Two scales have been used for assessing the level of enjoy in such groups: the Italian version of the Physical Activity Enjoyment Scale (PACES-IT) and the Enjoy Scale. The scales provided valid and interesting data for comparing the differences between the two groups. The obtained notes will be useful for supporting the work of the physical education teachers. Keywords: Physical activity; Children; Primary school; Enjoinment; Paces scale.
\end{abstract}

\section{Cite this article as:}

Pignato, S., Bonomo, R., Coppola, R., \& Casolo, F. (2019). An empirical study for assessing the level of enjoinment in an Italian primary school. Journal of Human Sport and Exercise, 14(4proc), S1017S1021. doi:https://doi.org/10.14198/jhse.2019.14.Proc4.63

\footnotetext{
Corresponding author. Faculty of Human and Society Sciences, University of Enna «Kore», Italy.

E-mail: salvatore.pignato@unikore.it

Supplementary Issue: Spring Conferences of Sports Science. Costa Blanca Sports Science Events, 14-15 June 2019. Alicante, Spain.

JOURNAL OF HUMAN SPORT \& EXERCISE ISSN 1988-5202

(c) Faculty of Education. University of Alicante

doi:10.14198/jhse.2019.14.Proc4.63
} 


\section{INTRODUCTION}

In Italy, several previous studies have addressed the level of motor competence in primary school children (Sgrò, Quinto, Pignato, Lipoma, 2016; Sgro, Quinto, Messana, Pignato, \& Lipoma, 2017; Sgrò, Mango, Pignato, Schembri, Licari, \& Lipoma, 2017; Sgrò, Quinto, Platania, \& Lipoma, 2019). Anyway, one of the main educational goals during childhood is related on the perceived enjoyment especially during physical activities. Indeed it is included in the physical education curriculum from the primary school to the secondary one, but also included in the different physical activities' projects in which it represent one of the main goals. Intrinsic motivation is positively related with the increase of the pleasure tested during the physical practice. The practice of motor and sports' activities could be representing an opportunity for enjoyment that allows those who practice it to overcome the difficulties of physical fatigue, to spent a large part of their free time to sports, to drop out others moments of leisure to the passion for sport and to the sacrifices it imposes on those who dedicate themselves to it with passion.

The motor and sport practice carried out with a methodological approach, care and personal satisfaction allows to remove the spectre of disaffection and the consequent drop out, one of the most serious problems that marks the divide between active and inactive population in our country.

Only in recent years in the international literature the enjoyment topic in sporting practice, the perception of the state of well-being that causes in determining the level of intrinsic motivation in maintaining constant levels of commitment in sports has been studied. One of the most famous and valid instruments to assess the enjoyment is the Physical Activity Enjoyment Scale (PACES) (Kendzierski, De Carlo, 1991). This scale was revised and update by Motl and collegues in 2001. In the 2008 was developed the Italian version of the Physical Activity Enjoyment Scale (PACES-it) that include 18 items (Carraro, Yang, Robbazza, 2008). In even more recent times the ENJOY SCALE was developed and it can be defined as non-specific scale with 25 items, with an additional means for assessing the levels of pleasantness applicable to disparate areas, including the sports one (Devidson, 2018).

\section{METHODS}

The following study involved a sample of 150 students ( 75 males and 75 females, average age: 9.45 years old) attending the fourth and fifth grades of two primary schools who had joined a national project carried out during the hours of physical education (i.e., CONI, "Sport di Classe").

Preliminarily, after to have acquired the parents' informal consent and the agreement of the headmaster, a cognitive test was administered. It is consisting in 5 thematic areas, with 56 questions to acquire information of the children and their parents about the habits and inclinations of sporting practice. The first part of the test consists of 12 questions necessary for the creation of the two groups of this study.

The first group (control group) included children who had not practiced any extracurricular sport activity, but only the activities carried out during the hours of physical education. The second group (experimental group) included children who practice structured sportive activity in informal environments (i.e. extra-school activities). Therefore the Physical Activity Enjoyment Scale (PACES) and the ENJOY Scale were administered to the sample. 
The aim of the following study is to determine the impact of motor/sport activity on the level of enjoyment through the two aforementioned scales (PACES and ENJOY) and to identify any group or gender differences in the perception of enjoyment.

The PACES, in detail, was used to verify the levels of enjoyment since it is considered a valid and reliable tool to see the level of pleasure of the movement practiced. However the PACES does not contain elements to understand the status of the acquisitions of the competences and/or abilities, the emotional, social and sharing component, the time factor as well as the environmental one (internal, external), elements that are, instead, fundamental and somewhat influential in the process of detecting the acquisition of the intensity of pleasure.

Therefore in support of the PACES scale the ENJOY scale was synergistically associated. It is made up onspecific scale with 25 items, with answers on a 7-point Likert scale marked by 5 subscales: pleasure, compatibility, competence, challenge/improvement, involvement.

The data obtained with the test and with the two scales were processed using the SPSS statistical software (IBM Statistical Package for Social Science). In agreement with the ethics committee of the University "Kore" of Enna, an informed consent was compiled by the parents and all students, to ensure the confidentiality of personal data, an alphanumeric identification code was assigned.

\section{RESULTS}

\section{PACES' scale results}

From the analysis of PACES' data positive results have emerged. High or medium-high scores were counted for voices related to questions about pleasure experienced during sport activity and low or medium-low scores for voices that are related to aspects that indicate less emotional-motivational involvement. These results indicate that the children enjoyed the following motor sports activity. The differences emerged between the fourth and fifth classes are few. The fifth grades show higher scores for positive voices and lower scores for negative voices than fourth graders pointing out that older pupils showed more fun than smaller ones during the proposed motor-sporting activity.

There are also differences between the control group and the experimental group. The experimental group shows higher scores in positive voices and lower scores in negative voices than in the control group. This indicates that the students in the experimental group had more fun than those in the control group. Moreover the results show a few gender differences. In detail the results show the female gender have appreciated more the physical activity practiced in the school gym than their male peers with the exception of two questions related to the motivational stimulation of the activity.

\section{ENJOY' scale results}

Positive results have been obtained from the ENJOY scale' data analysis. Were obtained high, medium-high results which indicate a high level of enjoyment. Only in two items were obtained average scores. In the results of the ENJOY scale there are slight differences between the fourth and fifth classes. In detail the fifth classes' children show highest values than fourth classes' children. In only four questions the situation is reversed and fourth grade children score are higher than fifth classes' children more. From this result, the fourth grade children' perception of the skills seems to be higher than the fifth ones and could be indicate that the latter underestimate their skills. 
The difference between the control and experimental group appears to be clear because when the latter shows higher scores compared to the control group, then the children of the experimental group enjoyed more than the children of the control group. Only in three items the control group has exceeded the experimental group. Furthermore, a statistically significant gender difference was found. The highest scores were achieved by the female in all items compared to male gender.

\section{DISCUSSION AND CONCLUSION}

The results obtained agree with previous studies (Carraro, Yang, Robazza, 2008; Devidson, 2018; Kendzierski, \& De Carlo, 1991; Motl et al.) and indicate a greater emotional involvement, during the motorsporting activity proposed, of older children (fifth classes) and the enjoyment increase in the experimental group that include children who also practice extracurricular activities. The PACES results relative to the gender difference, instead, are in disagreement with those that emerged from the studies conducted by Paxton and colleagues, which show a gendered invariance of the PACES demonstrated for children aged between 8 and 12 years (Paxton et al., 2008).

The reduced sample is the main limits of the research. Future studies can be directed to the enlargement of the sample, to the involvement of pupils with a wider age range, with a different social context of origin and with different levels of education.

It would also be necessary to increase the number of diversified training sessions with or/and without external variables and administer the questionnaires at the end of the training, to understand any differences in the enjoyment' perception.

These results and the methods used could be useful for the physical educational teacher to develop a physical educational program to develop not only the physical ability, but also to increase the enjoyment during the class schedule. The PACES and ENJOY scales used in this study could be used by the teacher to assess the enjoyment and the involvement of their pupils, and if necessary to change or modified the activities proposed during the hours of their teaching.

\section{REFERENCES}

Carraro A.,Valutare il piacere nelle attività motorie: il PACES-it, Italian Journal of Educazion, numero speciale, 2012, p. 259-264.

Carraro, A., Young, M. C., \& Robazza, C. (2008). A contribution to the validation of the physical activity enjoyment scale in an Italian sample. Social Behavior and Personality: an international journal, 36(7), 911-918. https://doi.org/10.2224/sbp.2008.36.7.911

Devidson S., A. (2018). Multi-Dimensional Model of Enjoyment: Development and Validation of an Enjoyment Scale (Enjoy), Embry Riddle Aereonautical University dissertation and tese Scolary Common Citation. http://commons.erau.edu/edt/381

Dunton, G., Tscherne J., Rodriguez, D., Validità fattoriale e invarianza di genere della scala di godimento dell'attività fisica (PACES) negli adolescenti anziani, Ricerca trimestrale per esercizio fisico e sport , v80 n.1 p.117-121, 2009.

Kendzierski D. e De Carlo K., Phisical Activity Enjoyment Scale: due studi di convalida, Journal of sport and Exercise Phychology, 1991, n. 13, p. 50-64. 
Sgrò, F., Quinto, A., Pignato, S., \& Lipoma, M. (2016). Comparison of product- and process-oriented model accuracy for assessing countermovement vertical jump motor proficiency in pre-adolescents. Journal of Physical Education and Sport. 16(3), 921-926.

Sgrò, F., Quinto, A., Messana, L., Pignato, S., \& Lipoma, M. (2017). Assessment of gross motor developmental level in Italian primary school children. Journal of Physical Education and Sport. 17(3), 1954-1959.

Sgrò, F., Mango, P., Pignato, S., Schembri, R., Licari, D., Lipoma, M. (2017). Assessing Standing Long Jump Developmental Levels Using an Inertial Measurement Unit. Perceptual and Motor Skills. Perceptual and Motor Skills, 124(1), 21-38. https://doi.org/10.1177/0031512516682649

Sgrò, F., Quinto, A., Platania, F., \& Lipoma, M. (2019). Assessing the impact of a physical education project based on sports-games approach on the actual motor competence of primary school children. Journal of Physical Education and Sport. 19(Supplement Issue 3), 781-786.

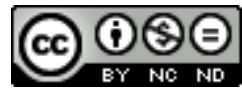

This work is licensed under a Attribution-NonCommercial-NoDerivatives 4.0 International (CC BY-NC-ND 4.0). 